\title{
A review of cardiac tumors
}

\section{Munasur, D. Reddy, A. Reddi and R. Kleinloog}

Department of Cardiothoracic Surgery, Inkosi Albert Luthuli Central Hospital and University of KwaZulu-Natal, Durban

\section{Address for correspondence:}

Darshan Reddy

Department of Cardiothoracic Surgery

Inkosi Albert Luthuli Central Hospital

Durban

4001

South Africa

Email:

darshanred@ialch.co.za

\section{ABSTRACT The intention of the review is to highlight} the clinical presentation and established surgical technique in the management of this relatively uncommon cardiac pathology.

Intracardiac masses may be encountered by a cardiologist or cardiac surgeon, and a comprehensive knowledge of the various diagnostic modalities is therefore essential. More crucial are the likely probabilities, based on an amalgamation of the clinical features, echocardiographic and radiographic findings. If surgical management is undertaken, the intra-operative finding is ultimately correlated with the histopathological diagnosis.

In the review, we emphasise the necessity of a multidisciplinary approach to management of these tumors. The cumulative experience of the various members of the team collectively enables increasingly accurate diagnoses to be made preoperatively,with appropriate surgical management carried out timeously. SAHeart 2008; 5:1 16-122

\section{INTRODUCTION}

In 1954 the first successful excision of a cardiac tumor was described. Utilising fibrillatory arrest via thoracotomy, Crafoord successfully resected a left atrial myxoma from a 40-year-old woman initially referred for atypical mitral stenosis.(1) Prior to the late 1950s, most cardiac tumors were discovered at postmortem examination. The development of echocardiography demonstrated the first echo diagnosis of an intracardiac tumor in 1959, thereby facilitating subsequent surgical referral and management.(2)
This historical perspective highlights two points of interest concerning the present-day diagnosis of cardiac tumors: the frequent clinical misdiagnosis as rheumatic heart disease, and the crucial role of echocardiography.

The high prevalence of rheumatic heart disease in South Africa accounts for the common misdiagnosis at primary care level of cardiac tumors as mitral valve pathology. Patients are initially investigated by echocardiography, a "screening" investigation with inherent operator dependent notoriety. The diagnosis of an intracardiac mass then provokes referral to a tertiary cardiology service for detailed investigation and subsequent surgical consideration.

The clinical incidence of primary cardiac tumors is about $\mid$ in 500 cardiac surgical cases and, with the exception of myxoma, will rarely be encountered in the career span of the average cardiologist or cardiothoracic surgeon. (I) The extensive use of imaging modalities such as CT scan and MRI in the investigation of unrelated disease, or in screening a "well patient", occasionally reveals an incidental cardiac tumor. $^{(3)}$ The radiographic characteristics of various cardiac tumors are well described and a few are illustrated in this review.

Surgery is often undertaken with a presumptive diagnosis predicated on the clinical presentation, echocardiographic and radiographic features. The intraoperative findings are often pathognomonic, with occasional exceptions. Histopathology and immunohistochemistry establishes the definitive answer and directs further management.

\section{LOCAL EXPERIENCE}

In the past 4 years, surgeons in the cardiothoracic unit at $I A L C H$ collectively excised 13 tumors within the pericardium. Tumor excision was occasionally combined with ancillary procedures, including mitral valve replacement, and nephrectomy. In addition, intracardiac masses encountered without excision included right ventricular thrombus, metastatic melanoma, neonatal rhabdomyoma and advanced bronchial carcinoma with left atrial invasion (Figure I).

Nine atrial myxomas were excised using a biatrial approach. Of note one myxoma was diagnosed incidentally, in a patient without cardiorespiratory symptoms, by CT scan for investigation of renal calculi. Four patients were mistaken clinically for rheumatic valvular disease, with revision to myxoma made on echocardiogram. 


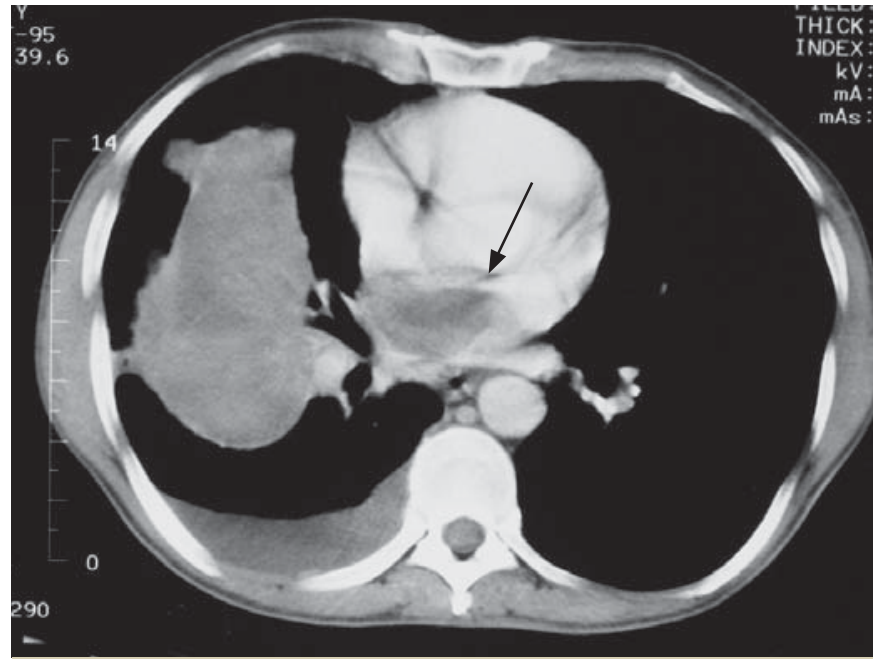

FIGURE I: Left atrial invasion (arrow) from bronchial carcinoma.

The following vignettes illustrate some of the protean modes of presentation and occasional diagnostic quandary associated with these uncommon tumors

\section{Case I}

A 33-year-old African female presented with intermittent swelling of the lower limbs and clinical features of significant mitral valve regurgitation, confirmed echocardiographically with leaflet thickening and calcification. Left ventricular contractility was good with an ejection fraction of $68 \%$, systolic and diastolic dimensions of $38 \mathrm{~mm}$ and $62 \mathrm{~mm}$ respectively. Coincidentally, a $4.2 \times 4.8 \mathrm{~cm}$ homogenous mass was seen in the right atrium, attached to the free wall. The tricuspid valve leaflets were normal with mild eccentric tricuspid regurgitation. Symptoms were ascribed to mitral regurgitation and necessitated surgery.

At operation, on opening the pericardium, the mass was easily visualized and thought to be a well encapsulated lipoma arising from the right atrial free wall (Figure 2) and suitable for excision with a cuff of right atrium (Figure 3) and direct suture closure of the defect. Via a left atriotomy the mitral valve was replaced with a mechanical prosthesis. Histology confirmed a completely resected encapsulated lipoma (Figure 4) and the valve showed features of chronic rheumatic valvulitis, with no evidence of infective endocarditis.

\section{Case 2}

A 2-day-old neonate was referred to the tertiary neonatal intensive care unit following mechanical ventilation for progressively worsening respiratory distress 6 hours after caesarian section for prolonged labour, with APGAR scores of 8 and 9 at one and five minutes respectively.

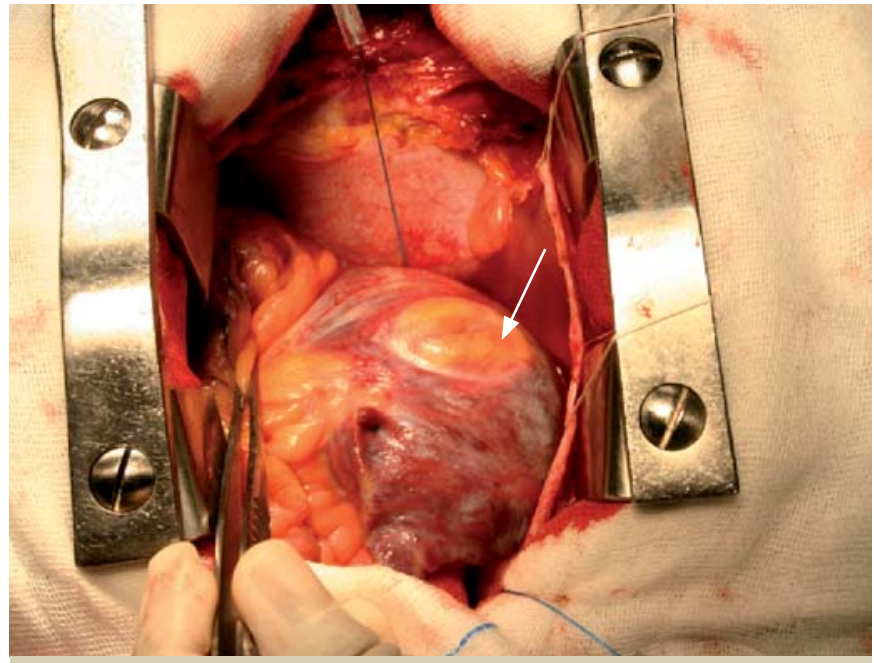

FIGURE 2: Right atrial lipoma (arrow) in situ.

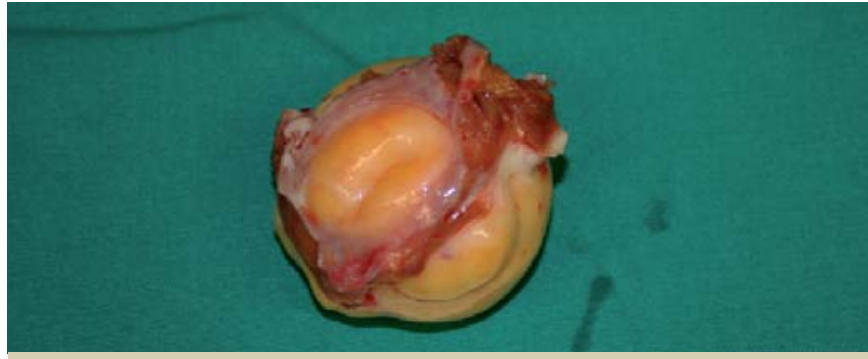

FIGURE 3: Lipoma excised with margin of right atrial free wall.

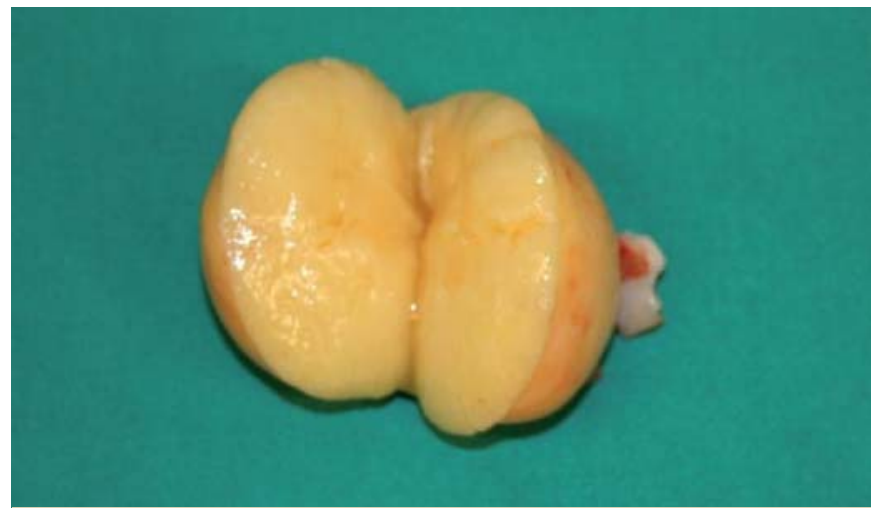

FIGURE 4: Cut section of encapsulated cardiac lipoma.

Antenatal routine foetal ultrasound had detected fluid within the thoracic cavity without clear visualization of the 4 cardiac chambers. Plain chest radiography on Day I showed a markedly enlarged mediastinal silhouette (Figure 5), and echocardiography confirmed a pericardial effusion $(20 \mathrm{~mm}$ anteriorly, $13 \mathrm{~mm}$ posteriorly and $5 \mathrm{~mm}$ apically) and a large intrapericardial mass. A CT scan was done for further elucidation (Figure 6). This showed a large, homogenous soft 


\section{CARDIAC TUMORS}

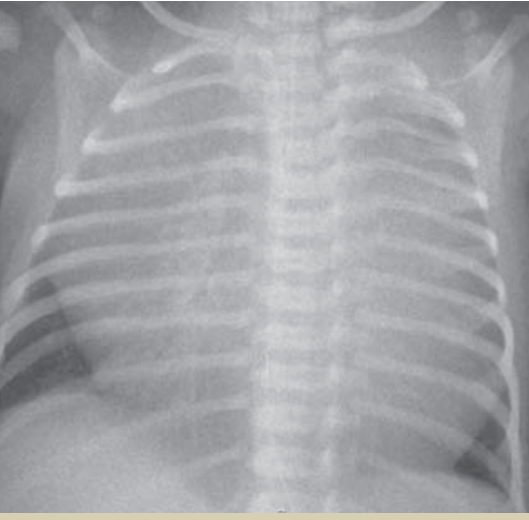

FIGURE 5: Markedly enlarged cardiac silhouette.

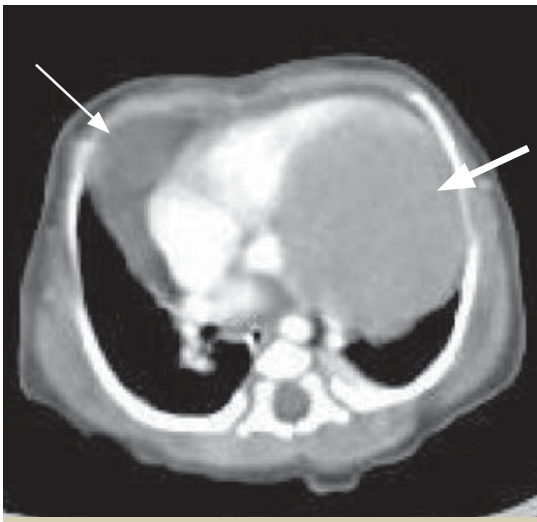

FIGURE 6: Intrapericardial mass (large arrow) with associated pericardial effusion (small arrow).

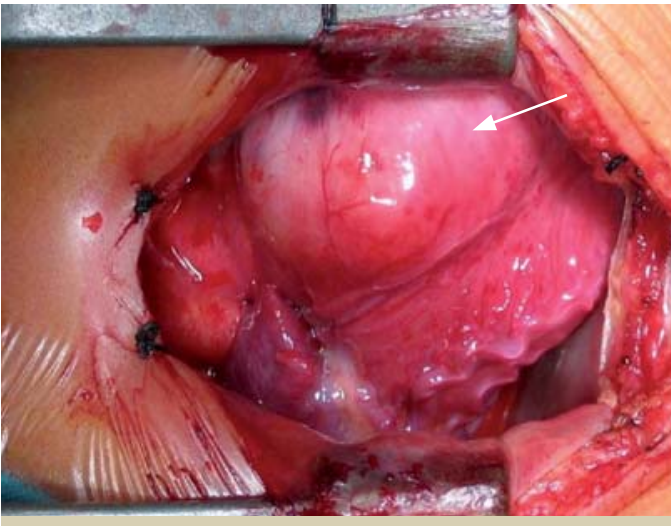

FIGURE 7: Rhabdomyoma (arrow) arising within ventricular muscle.
GURE 7.Rhabdomyoma (arrow) arising within ventricular muscle. tissue mass within the pericardium measuring $53 \mathrm{~mm} \times 41 \mathrm{~mm}$. At operation, via median sternotomy, in addition to the serous pericardial collection, a large homogenous soft tissue mass arising from the anterior myocardium of the left ventricle was encountered (Figure 7). Attempts at dissection of the tumor proved futile. The procedure was therefore abandoned in favour of multiple core needle biopsies. Histopathology and immunohistochemistry were consistent with a cardiac rhabdomyoma. She is currently being investigated for tuberous sclerosis, known to be associated with cardiac rhabdomyomas. ${ }^{(4)}$

\section{Case 3}

A 4-year-old child with stage IV nephroblastoma was referred for excision of residual tumor within the IVC and right atrium. The child had undergone right nephrectomy 4 weeks previously and had been commenced on chemotherapy in the interim. CT scan prior to nephrectomy demonstrated the large right renal mass with right atria extension of tumor (Figure 8). Echocardiography showed tumor (measuring $48 \mathrm{~mm} \times 33 \mathrm{~m}$ ) within the inferior vena cava extending into the right atrium. Surgery was undertaken with deep hypothermic ( 16 degrees centigrade) circulatory arrest. Via the right atrium residual tumor, densely adherent to the interatrial septum and IVC, was dissected and excised. Complete excision of IVC and right atrial tumor was confirmed with a post operative CT scan. Histology was consistent with metastatic nephroblastoma.

\section{Case 4}

A 14-year-old male was referred from the Department of Oncology with congestive cardiac failure. Two years previously he had undergone an above-knee amputation for osteogenic sarcoma and was currently on chemotherapy via an indwelling central venous catheter (Groshong line). Plain chest radiography demonstrated bilateral pleural collections and an enlarged cardiac silhouette, in keeping with congestive cardiac failure. Aspiration revealed serous effusions with no malignant cells. Echocardiogram demonstrated a large right ventricular mass, illustrated by $\mathrm{CT}$ scan of the thorax and thought to represent a thrombus intimately associated with the central venous catheter (Figure 9). The patient declined intervention

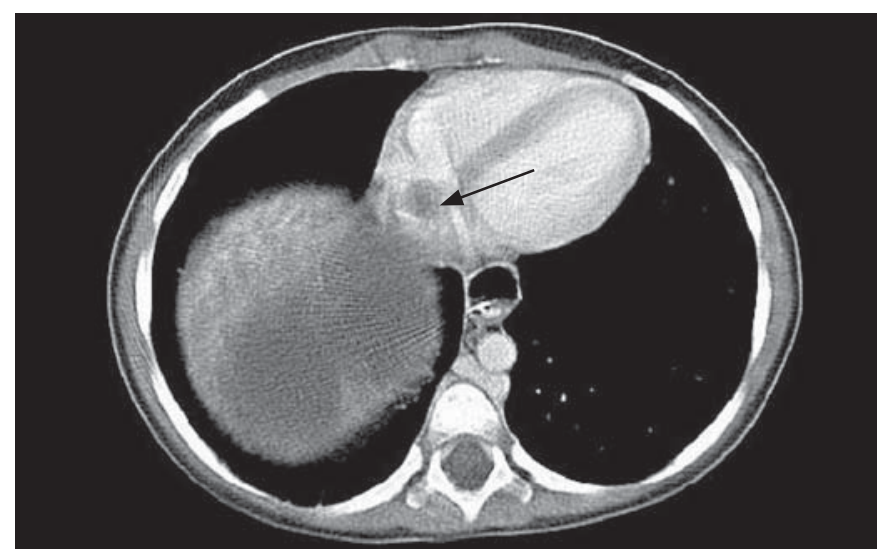

FIGURE 8: Right atrial spread of nephroblastoma (arrow).

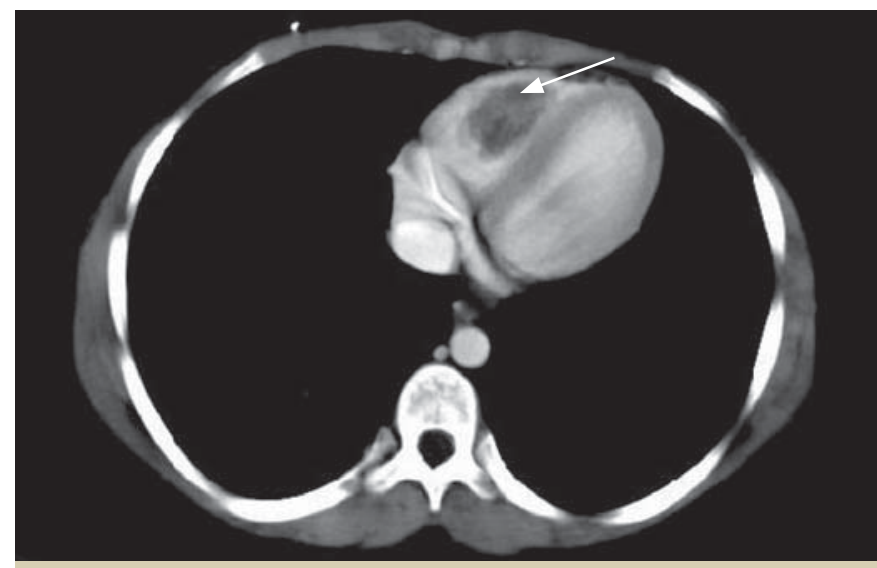

FIGURE 9: Right ventricular mass (arrow) associated with long-term indwelling central venous line. 


\section{PATHOLOGICAL CLASSIFICATION}

Cardiac tumors may be classified as primary (benign or malignant) or secondary neoplasms that have metastasized to the heart. The incidence of primary cardiac neoplasms in random autopsy series ranges between $0.17-0.19 \%{ }^{(5)}$ Secondary involvement of the heart or pericardium occurs in 10-20\% of patients with disseminated cancer. ${ }^{(6)}$ In addition to metastases, contiguous spread from pulmonary or mediastinal tumors may result in invasion of intrapericardial structures.

Approximately $75 \%$ of primary cardiac tumors are benign, with half of this group comprising myxomas. Myxomas are the commonest primary cardiac tumors. ${ }^{(7)}$ In infants and children, the most common primary cardiac tumor is a rhabdomyoma. Sarcomas make up 75\% of primary malignant tumors, with angiosarcoma being commonest. ${ }^{(8)}$

Primary cardiac tumors *

\begin{tabular}{|c|c|}
\hline \multicolumn{2}{|c|}{ Infancy and childhood (<16 years old) } \\
\hline Benign & Malignant \\
\hline Rhabdomyoma & Rhabdomyosarcoma \\
\hline Fibroma & Angiosarcoma \\
\hline Myxoma & Malignant fibrous histiocytoma \\
\hline Hemangioma & Leiomyosarcoma \\
\hline Mesothelioma of AV node & Fibrosarcoma \\
\hline Purkinje cell tumor & Myxosarcoma \\
\hline Teratoma & Unclassified \\
\hline \multicolumn{2}{|c|}{ Adult } \\
\hline Benign & Malignant \\
\hline Myxoma & Angiosarcoma \\
\hline Papillary fibroelastoma & Malignant fibrous histiocytoma \\
\hline Hemangioma & Osteosarcoma \\
\hline Lipomatous hypertrophy (atrial septum) & Leiomyosarcoma \\
\hline Mesothelioma of AV node & Fibrosarcoma \\
\hline Fibroma & Myxosarcoma \\
\hline Lipoma & Rhabdomyosarcoma \\
\hline Paraganglioma & Synovial sarcoma \\
\hline \multirow[t]{3}{*}{ Miscellaneous } & Liposarcoma \\
\hline & Malignant schwannoma \\
\hline & Unclassified \\
\hline
\end{tabular}

*Adapted from: Tumors of the heart and pericardium, Diagnostic histopathology of tumors 2nd ed, Ch.D.M. Fletcher.

Secondary cardiac tumors

\begin{tabular}{|l|l|}
\hline & \multicolumn{1}{c|}{ Malignant } \\
\hline Melanoma & Renal cell carcinoma \\
Sarcoma & Nephroblastoma \\
& Bronchial carcinoma \\
\hline
\end{tabular}

\section{MYXOMA}

This entity merits special attention as it is the commonest cardiac neoplasm, representing $50 \%$ of all benign cardiac tumors. ${ }^{(9)}$ The peak incidence is between the 3rd and 6th decades, with a female preponderance. ${ }^{(10)}$

Myxomas arise from the endocardial mesenchyme and consist of a polysaccharide matrix made up of smooth muscle, collagen and elastin. ${ }^{(1)}$ The macroscopic appearance is either gelatinous, lobulated or smooth. (12) They may manifest in sessile, polypoid or pedunculated form. Approximately two-thirds of myxomas are polypoid, with a smooth, lobulated surface and are unlikely to break off spontaneously (Figure 10). (13) The remainder, by contrast, are of the villous variety with a gelatinous consistency, and a propensity for fragmentation and embolization (Figure II). In addition to an overall firm consistency some myxomas demonstrate gross calcification, which may form the bulk of the tumor. This condition is referred to as "petrified cardiac myxoma" and may be mistaken for an atrial thrombus. ${ }^{(7)}$

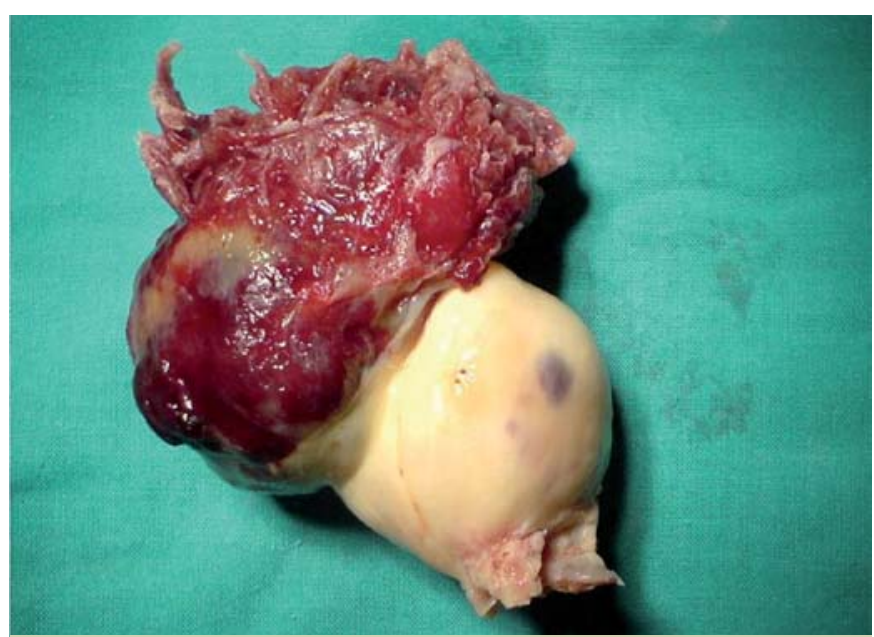

FIGURE 10: Polypoid, lobulated myxoma with adherent fibrin. This "solid" variety is less likely to fragment spontaneously.

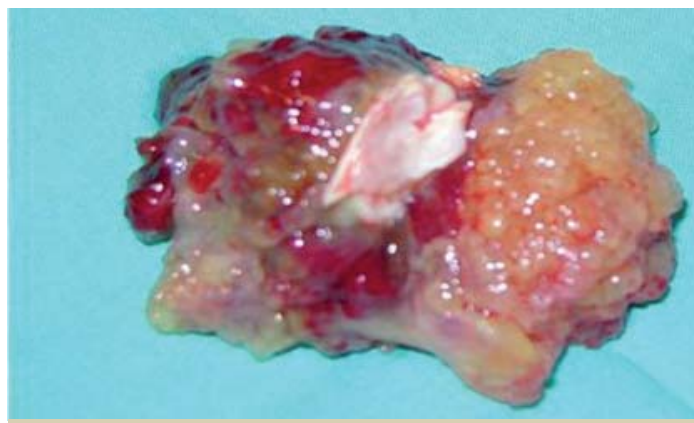

FIGURE I I:Villous, gelatinous myxoma with increased propensity for fragmentation and distal embolization. 


\section{CARDIAC TUMORS}

While the overwhelming majority of myxomas occur sporadically, 5\% have a familial pattern based on autosomal dominant inheritance. ${ }^{(14)}$ The familial type often afflicts the young with equal gender distribution, and is often multicentric with a higher recurrence rate after surgery. ${ }^{(15)}$

Myxomas may originate in any chamber: $75 \%$ occur in the left atrium and 10-20\% in the right. ${ }^{(16)} 94 \%$ of myxomas are solitary. They are known to arise from any part of the atrium including the atrial appendage. Most arise from the interatrial septum at the border of the fossa ovalis. Generally, right atrial myxomas have broad-based attachments. Right ventricular lesions arise from the ventricular free wall whilst left ventricular myxomas arise from the posterior papillary muscle. $^{(17)}$

\section{CLINICAL PRESENTATION OF CARDIACTUMORS}

As cardiac tumors are slow-growing neoplasms, most patients remain asymptomatic until the tumor has reached sufficient proportion to cause complications. Incidental tumors are often detected during the investigation of other cardiac disease. Patients with cardiac tumors may be asymptomatic or present with constitutional symptoms, palpitations, congestive cardiac failure, thromboembolism or infection.

The common constitutional features are: loss of weight, fever, lethargy, clubbing, arthralgia and myalgia. These may occur in association with hematological abnormalities including leucocytosis, increased erythrocyte levels and sedimentation rate, hemolytic anemia, thrombocytopenia, elevated C-reactive protein, increased circulating immunoglobulin $\mathrm{G}$ and interleukin-6.(18)

Obstruction of cardiac blood flow is determined by the size of the tumor as well as the chamber involved. Obstructive lesions in the left atrium tend to mimic mitral valve disease, predominantly mitral stenosis. Obversely, mitral regurgitation occurs when a voluminous tumor interferes with mitral leaflet coaption. The clinical presentation may be that of cardiac failure with elevated left atrial and pulmonary venous pressures. Obstructive lesions in the right atrium simulate tricuspid valve stenosis and may produce the clinical features of right ventricular failure. In the presence of a patent foramen ovale, right-to-left atrial shunting may occur with ensuing central cyanosis and the potential for paradoxical embolization. Ventricular tumors may cause outflow obstruction, mimicking subaortic, aortic or pulmonary valve stenosis.

Systemic embolization occurs in $30-40 \%$ of patients with atrial myxomas. ${ }^{(19)}$ With left-sided tumors, the majority of embolic episodes affect the central nervous system. Other areas include the lower limb, pulmonary, abdominal visceral, renal and coronary artery circulations.

Infection occurring within a cardiac tumor is a rare phenomenon. The clinical picture resembles infective endocarditis and the increased risk of tumor embolisation constitutes an indication for urgent surgery.

\section{DIFFERENTIAL DIAGNOSIS}

The clinical features of an intracardiac mass causing interference with valvular function may simulate rheumatic valvular disease or infective endocarditis.

The intrapericardial component of a bronchial or mediastinal tumor may be misinterpreted as a cardiac tumor if investigated initially by echocardiography for cardiac symptoms. A mural thrombus may mimic an intracardiac mass both clinically and radiographically.(16) Mural thrombi are associated with myocardial infarction, atrial fibrillation with or without valve disease, long-term use of central venous catheters and hypercoaguable states.

\section{INVESTIGATIONS}

\section{Chest radiograph}

The radiograph may appear normal or show features of abnorma cardiac silhouette, chamber enlargement, pulmonary congestion and congestive cardiac failure. Occasionally, calcification may be visible in the tumor, particularly right-sided myxomas.

\section{Electrocardiogram}

The electrocardiographic findings are usually nonspecific and include chamber enlargement, cardiomegaly, bundle-branch block and axis deviation.

\section{Echocardiogram}

The transthoracic echocardiogram usually provides adequate diagnostic information for surgical resection and is sufficiently sensitive to predict the etiology of most intracavitary masses, especially myxoma. The transoesophageal echocardiogram provides superior definition of tumor size, location, mobility and attachment and is also useful to evaluate the posterior left atrial wall, interatrial septum and the right atrium. ${ }^{(18)}$ Intraoperative transoesophageal echocardiography also provides a useful means of confirming adequate tumor resection. 


\section{Computed tomography}

The use of computed tomography is greatest in the evaluation of malignant cardiac tumors because of its ability to demonstrate myocardial and extramyocardial tumor invasion.

\section{Magnetic resonance imaging (MRI)}

This modality provides high-definition images of soft-tissue tumors and hence, may yield a comprehensive picture of tumor size, shape and surface characteristics. In addition, MRI is useful in detecting intracardiac and pericardial extension and invasion by malignant secondary tumors. ${ }^{(21)}$

\section{INDICATIONS FOR SURGERY}

The diagnosis of a primary cardiac tumor confined to the heart is, per se, an indication for surgery. The timing of intervention is influenced by the presence of complications such as obstruction to cardiac blood flow, embolization and infection, mandating emergency surgery. An estimated $8 \%$ of patients die while awaiting surgery. ${ }^{(22)}$

\section{SURGICAL STRATEGY}

The heart and great vessels are customarily and best approached via a median sternotomy. During cannulation of the ascending aorta and vena cavae, it is vital to avoid cardiac manipulation to minimize the risk of precipitating tumor embolization.

For left atrial tumors, the vena cavae are cannulated via the right atrium with the inferior vena caval cannula placed close and lateral to the inferior vena cava/right atrial junction.

When a malignant left atrial tumor is suspected, or if extensive exposure is necessary, then the superior vena cava is cannulated directly. This permits transection of the superior vena cava for additional exposure.

Right atrial tumors carry special cannulation considerations in view of the risk of tumor fragmentation and dislodgement. Both vena cavae may be cannulated directly. CT scan or MRI is useful to determine the extent of intravascular tumor spread as the presence of low or highlying tumor may necessitate cannulation of the femoral or jugular veins

Cardiopulmonary bypass with mild to moderate hypothermia is instituted and the aorta cross-clamped prior to cardioplegic delivery.
Atrial tumors may be approached either via the chamber of origin directly (atrial approach) or via a biatrial approach for tumors attached to the interatrial septum. ${ }^{(23)}$ Entry into multiple chambers necessitates meticulous cardiac deairation prior to aortic declamping.

Left atrial tumors may be approached via a standard transverse incision anterior to the right pulmonary veins. Exposure of large tumors attached to the interatrial septum may be facilitated by a parallel counter-incision in the right atrium, enabling the surgeon to locate the septal attachment. Left atrial tumors arising from the interatrial septum are also amenable to resection via a right atriotomy and trans-septal approach.

Ventricular tumors may be approached through the atrio-ventricular valve, which may be detached anteriorly to improve exposure. Access may also be obtained via a transvalvular approach or ventriculotomy. ${ }^{(24)}$

In principle, it is desirable to resect the tumor en bloc without fragmentation as well as a full-thickness margin of the surrounding myocardium or septum to which it is attached. The full-thickness defect is then reconstructed with either a patch of autologous pericardium or synthetic material. In practice, only a partial thickness resection may be possible due to anatomical constraints such as underlying conduction tissue or valvular structures, accepting a concomitant increased risk of tumor recurrence. Recurrence is also postulated to be a result of incomplete atrial septectomy for tumors arising from the interatrial septum. ${ }^{(25)}$

Advanced surgical techniques for the excision of relatively inaccessible, locally invasive malignant tumors, such as malignant fibrous histiocytoma, include explantation of the heart with ex vivo aggressive resection and reconstruction of the left atrium. This technique has produced satisfactory results in experienced centres. ${ }^{(9)}$

Following tumor excision, copious irrigation of the relevant cardiac chamber with evacuation of residual tumor fragments via the wall suction is essential to prevent systemic embolisation. The adjacent atrio-ventricular valve is then inspected for damage to the annulus, leaflets or chordae, mindful of advocacy of valve repair and preservation. Should a multicentric tumor be suspected, then all four cardiac chambers must be inspected and complete resection confirmed with transesophageal echocardiography. 


\section{CARDIAC TUMORS}

The utility of an intraoperative transoesophageal echocardiogram lies in the evaluation of the lesion, facilitation of safe cannulation, confirmation of complete tumor removal and assessment of the adequacy of repair and cardiac deairation. ${ }^{(26)}$

\section{LONG-TERM ECHOCARDIOGRAPHIC SURVEILLANCE}

Long-term echocardiographic surveillance is indicated in the following instances: tumors that are multicentric or present in unusual cardiac locations, incomplete tumor resection, abnormal tumor DNA genotype and myxoma with malignant histological characteristics. ${ }^{(24)}$

\section{RESULTS}

The operative mortality for benign tumors is less than $5 \%,{ }^{(10)}$ with a I$4 \%$ recurrence rate for myxomas of the sporadic type and a $22 \%$ recurrence rate for the familial type.(II) Recurrence rates and long-term survival for malignant tumors are contingent upon pathological tumor stage and biological characteristics of the tumor.

\section{CONCLUSION}

Interdepartmental collaboration for assessment of cardiac tumors is obligatory. Key members of the management team include a cardiologist, radiologist, cardiac surgeon and pathologist. The use of enhanced radiographic imaging, intraoperative TEE and professional surgical photography promote interaction at various levels.

A team approach leads to improved clinicopathological correlation and permits utilization of the available knowledge and skills. If maximal information is extracted and analyzed from individual cases, the unit is able to collectively accumulate expertise on the local nuances of an uncommonly encountered condition.

The unfortunate reality is that ancillary members tend to surreptitiously fall by the wayside (unless, of course, there is pecuniary interest) as the patient is wheeled into the operating room, and often it is only the surgical team that gains maximal exposure, including figuratively, to the pathology.

\section{REFERENCES:}

I. Chitwood WR Jr. Clarence Crafoord and the first successful resection of a cardiac myxoma. Ann Thorac Surg 1992;54:997-8.

2. Effert S, Domanig E. Diagnostik intraaurikularerTumoren an grosserThromben mit dem Ultraschall-Echoverfahren. Dtsch Med Wochesch 1959;84:6.

3. Perchinsky MJ, Lichtenstein SV, Tyers GF. Primary cardiac tumors. Cancer. 1997;79:9.

4. Yu, et al. Epidemiological and pathological characteristics of cardiac tumors: a clinical study of 242 cases. Interact CardioVasc Thorac Surg 2007;6:636-639.

5. Wold LE, Lie JT. Cardiac myxomas: A clinicopathologic profile. Am J Pathol 1980; 101:219.

6. Smith C. Tumors of the heart. Arch Pathol Lab Med 1986; 1 10:371

7. Becker AE. Tumors of the heart and pericardium. Ch.D.M. Fletcher Diagnostic histopathology of tumors: 2nd ed. London: Churchill Livingstone, 2000.

8. Silverman NA. Primary cardiac tumors. Ann Surg 1980; 91:127.

9. Walkes J-CM, Smythe WR, Reardon MJ. Cardiac Neoplasms. Cohn L, ed. Cardiac Surgery in the Adult. New York: McGraw-Hill, 2008.

10. Pinede L, Duhaut P, Loire R. Clinical presentation of left atrial cardiac myxoma: A series of I I 2 consecutive cases. Medicine 200 I;80:159

I I. Reynen K. Cardiac myxomas. N Engl J Med 1995;333:1610.

12. Prichard RW. Tumors of the heart: Review of the subject and report of one hundred and fifty cases. Arch Pathol 1951; 51:98.

13. McAllister HA, Fenoglio JJ Jr. Tumors of the cardiovascular system, in Atlas of Tumor Pathology, Series 2. Washington, Armed Forces Institute of Pathology, 1978.

14. Gelder HM, O'Brian DJ, Styles ED, et al. Familial cardiac myxoma. Ann Thorac Surg 1992; 53:419.

15. Farrah MG. Familial cardiac myxoma: Study of patients with myxoma. Chest 1994; 105:65

16. Waller R, Grider L, Rohr T, et al. Intracardiac thrombi: Frequency, location, etiology and complications: A morphologic review, part I. Clin Cardiol 1995; I 8:477.

17. Imperio J, Summels D, Krasnow N, et al. The distribution patterns of biatrial myxoma Ann Thorac Surg 1980;29:469.

18. Kuroki S, et al. Increased interleukin-6 activity in cardiac myxoma with mediastinal lymphadenopathy. Intern Med 1992;31:1207.

19. Goodwin JF. The spectrum of cardiac tumors. Am J Cardiol 1968;21:307.

20. Ensberding R, Erbel DR, Kaspar W, et al. Diagnosis of heart tumors by transesophagea echocardiography. Eur Heart J 1993; | 4: 1223.

21. Case Records of the Massachusetts General Hospital, Weekly Clinicopathological Exercises: Case 14-1978. N Engl J Med 1978;298:834

22. Nkere UU, Pugsley WB. Time relationships in the diagnosis and treatment of left-atria myxoma. Thorac Cardiovasc Surg 1993;41:301.

23. Stevens LM, et al. Atrial versus biatrial approaches for cardiac myxomas. Interact CardioVasc Thorac Surg 2003:2:521-525.

24. Dato GMA, Benedictus M, Dato AA, et al. Long-term follow-up of cardiac myxomas (7-31 years). J Cardiovasc Surg 1993;34:141.

25. Bertolotti U, Mazzucco A, Valfre C, et al. Right ventricular myxoma: Review of the literature and report of two patients. Ann Thorac Surg 1983;33:277.

26. Reeder GS, Khandheria BK, Senard JB, et al. Transesophageal echocardiographs and cardiac masses. Mayo Clin Proc 1991;66:1 I01. 